University of Nebraska - Lincoln

DigitalCommons@University of Nebraska - Lincoln

Timothy J. Gay Publications

Research Papers in Physics and Astronomy

2006

\title{
Angular Momentum Partitioning in the Dissociation of Diatomic Molecules
}

Timothy J. Gay

University of Nebraska - Lincoln, tgay1@unl.edu

J. D. Bozek

Advanced Light Source, Lawrence Berkeley Laboratory

J. E. Furst

University of Newcastle-Ourimbah, Ourimbah, NSW

Gordon A. Gallup

University of Nebraska - Lincoln, ggallup1@unl.edu

A. S. Green

University of St. Thomas, St. Paul, MN

See next page for additional authors

Follow this and additional works at: https://digitalcommons.unl.edu/physicsgay

Part of the Physics Commons

Gay, Timothy J.; Bozek, J. D.; Furst, J. E.; Gallup, Gordon A.; Green, A. S.; Kilcoyne, A. L. D.; Machacek, J. R.; Maseberg, J. W.; McLaughlin, K. W.; and Rosenberry, Mark A., "Angular Momentum Partitioning in the Dissociation of Diatomic Molecules" (2006). Timothy J. Gay Publications. 53.

https://digitalcommons.unl.edu/physicsgay/53

This Article is brought to you for free and open access by the Research Papers in Physics and Astronomy at DigitalCommons@University of Nebraska - Lincoln. It has been accepted for inclusion in Timothy J. Gay Publications by an authorized administrator of DigitalCommons@University of Nebraska - Lincoln. 


\section{Authors}

Timothy J. Gay, J. D. Bozek, J. E. Furst, Gordon A. Gallup, A. S. Green, A. L. D. Kilcoyne, J. R. Machacek, J. W. Maseberg, K. W. McLaughlin, and Mark A. Rosenberry 


\title{
Angular Momentum Partitioning in the Dissociation of Diatomic Molecules
}

\author{
T.J.Gay*, J.D.Bozek ${ }^{\dagger}$, J.E.Furst ${ }^{\ddagger}$, G.A.Gallup*, A.S.Green**, \\ A.L.D.Kilcoyne ${ }^{\dagger}$, J.R.Machacek*, J.W.Maseberg*, K.W.McLaughlin", \\ and M.A.Rosenberry*\$ \\ *Behlen Laboratory of Physics, University of Nebraska, Lincoln, NE 68588-0111 USA \\ ${ }^{\dagger}$ Advanced Light Source, Lawrence Berkeley Laboratory, Berkeley, CA 94720, USA \\ ${ }^{7}$ University of Newcastle-Ourimbah, Ourimbah, NSW 2258, Australia \\ ${ }^{+}$University of St. Thomas, 2115 Summit Ave., St. Paul, MN 55105-1080 USA \\ ${ }^{\#}$ Loras College, Department of Physics and Engineering, Dubuque, IA 52001 USA \\ ${ }^{8}$ Siena College, Department of Physics, Loudonville, NY 12211 USA
}

\begin{abstract}
We discuss recent experiments that study the transfer of angular momentum from a projectile to the residual target in collisions between the simple diatomic molecules $\mathrm{H}_{2}$ and $\mathrm{N}_{2}$ and spin-polarized electrons or circularly-polarized photons. We observe the fluorescence of both the atomic fragments and excited molecular states, and measure the circular polarization fraction of this light, $\mathrm{P}_{3}$. The incident electron energies range from 10 to $100 \mathrm{eV}$; the incident photon energies from 33 to $38 \mathrm{eV}$.
\end{abstract}

Keywords: angular momentum; spin polarization; circular polarization; molecular dissociation PACS: 33.15.Vb; 33.80.Gj; 34.30. $+\mathrm{h} ; 34.80 . \mathrm{Ht} ; 34.80 . \mathrm{Nz}$

\section{INTRODUCTION}

An important problem in atomic collisions is the distribution, or partitioning, of angular momentum in an excited or ionized atomic target produced by photon or electron bombardment [1]. A complete picture of the angular momentum dynamics must include the ionized or scattered electrons as well [2]. An interesting extension of this problem involves molecular targets, which have the additional complication of rotational angular momentum. One experimental approach to the general problem of angular momentum dynamics in such collisions is to use incident photon or electron beams that are spin polarized, and to detect the polarization of the fluorescence emitted by the target or its fragments following the collision. The advent of thirdgeneration light sources and GaAs polarized electron sources has made such experiments much easier. Earlier experiments had used unpolarized electrons and detected the polarization (both circular and linear) of emitted light in coincidence with the scattered electron $[3,4]$, or used linearly-polarized incident light and detected linear polarization of the subsequent molecular or atomic fluorescence[5-7]. We report here the results of several recent experiments done by our group and others which have begun to elucidate how angular momentum, inserted into the collision complex by the use of either polarized electrons or photons, is distributed in a collision with a simple 
diatomic molecule or molecular ion. We will concentrate on the measurement of $\mathrm{P}_{3}$, the circular polarization fraction (or Stokes parameter), as a direct measure of the angular momentum transferred along a specific axis to the target by the incident polarized particle.

\section{EXPERIMENTS WITH ELECTRONS}

A particularly simple example of angular momentum transfer in the electronimpact excitation of atoms is one in which a longitudinally-polarized electron excites, via exchange, an upper state of the atom which subsequently emits a photon that is circularly polarized along the axis of the incident electron. Ultimately, for an atom to emit circularly-polarized light, its orbital angular momentum must have a magnetic dipole along the direction of photon emission. The incident electron spin provides this through the atom's internal spin-orbit coupling. An example of this occurs in the exchange excitation of the $1 \mathrm{~s} 3 \mathrm{p} 3^{3} \mathrm{P}$ state of He. For $100 \%$ incident electron spin polarization, the emitted photon in the $2^{3} \mathrm{~S}-3^{3} \mathrm{P} 389 \mathrm{~nm}$ transition has a $\mathrm{P}_{3}$ of $50 \%$ near threshold [8]. Similar angular momentum transfer has been observed in a variety of atomic systems $[9,10]$.

Problems arise with molecules, however. The Münster group bombarded $\mathrm{N}_{2}$ in its singlet closed-shell ground state with beams of polarized electrons, and measured $\mathrm{P}_{3}$ of the resultant $C^{3} \Pi_{u}-B^{3} \Pi_{g} 337 \mathrm{~nm}$ fluorescence [11]. The excitation of a triplet state with light targets guarantees that exchange excitation, and thus angular momentum transfer, has occurred. Within their statistical uncertainty of $2 \times 10^{-3}$, however, they found $\mathrm{P}_{3}$ to be nil at all the incident electron energies they investigated (see Figure 1).

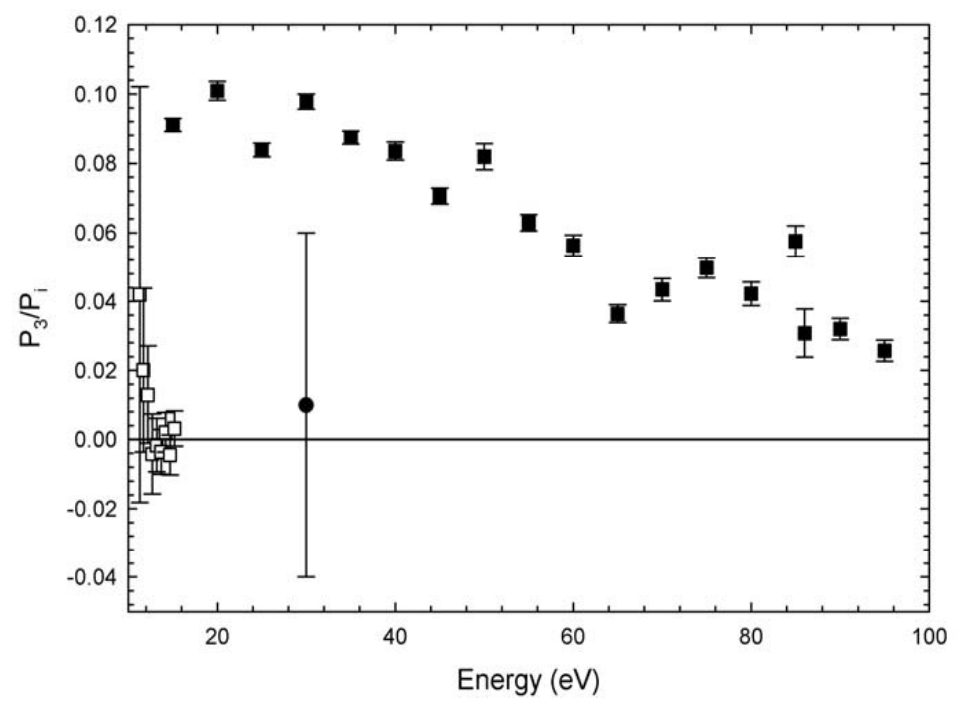

FIGURE 1. Circular polarization fraction $\mathrm{P}_{3}$ normalized to incident electron polarization $v s$. incident electron energy. Open squares: data of ref. 11; solid circle: measurement with $\mathrm{N}_{2}$ target and $388 \mathrm{~nm}$ filter (see text); solid squares: data of ref. 12 taken with a $600 \pm 5 \mathrm{~nm}$ filter. 
A similar, more crude measurement was recently made in our lab, in which we used an interference filter with a bandpass of $388 \pm 5 \mathrm{~nm}$. This filter isolates light from the $A^{3} \Sigma_{u}^{+}-X^{1} \Sigma_{g}^{+}, \quad C^{3} \Pi_{u}-B^{3} \Pi_{g}, \quad A^{3} \Sigma_{u}^{+}-X^{1} \Sigma_{g}^{+}, \quad C^{3} \Pi_{u}-B^{3} \Pi_{g}, \quad$ and $\quad C^{\prime 3} \Pi_{u}-B^{3} \Pi_{g}$ transitions in $\mathrm{N}_{2}$, and the $B^{2} \Sigma_{u}^{+}-X^{2} \Sigma_{g}^{+}$transitions in $\mathrm{N}_{2}^{+}$. Since the $\mathrm{N}_{2}$ transitions involve triplet states, and the $\mathrm{N}_{2}^{+}$excited state is a doublet, exchange collisions dominate the production of the light we observe. Nonetheless, as with the Münster data, we find $\mathrm{P}_{3}$ to be consistent with zero (Fig. 1).

We can start to understand these data by remembering the relevant time scales for molecular processes. The impact excitation occurs in times of the order of $10^{-16} \mathrm{~s}$, whereas $\mathrm{N}_{2}$ rotational motion occurs on a scale of $\sim 10^{-13} \mathrm{~s}$, with fluorescence lifetimes being more typically $10^{-8} \mathrm{~s}$. The spin-orbit coupling time for excited states of $\mathrm{N}_{2}$, i.e., the time required for the electron to "spin-up" the orbital angular momentum of the excited state, is $\sim 10^{-13} \mathrm{~s}$. Thus while the $\mathrm{N}_{2}$ target develops an orbital orientation over the course of several rotational periods, its internuclear axis subsequently rotates thousands of times before it decays, causing its orbital orientation to be essentially randomized in space. Thus $\mathrm{P}_{3}$ is nil.

One can, however, expect that an exchange collision followed by a prompt dissociation of the molecule would result in atomic fragments with a non-zero expectation value of spin along the incoming electron axis. The atomic "memory" of this spin direction would not be lost. Thus it should be possible to investigate angular momentum transfer to the molecular fragments by investigating the circular polarization of atomic fluorescence. Using this idea, our group and the Perth group

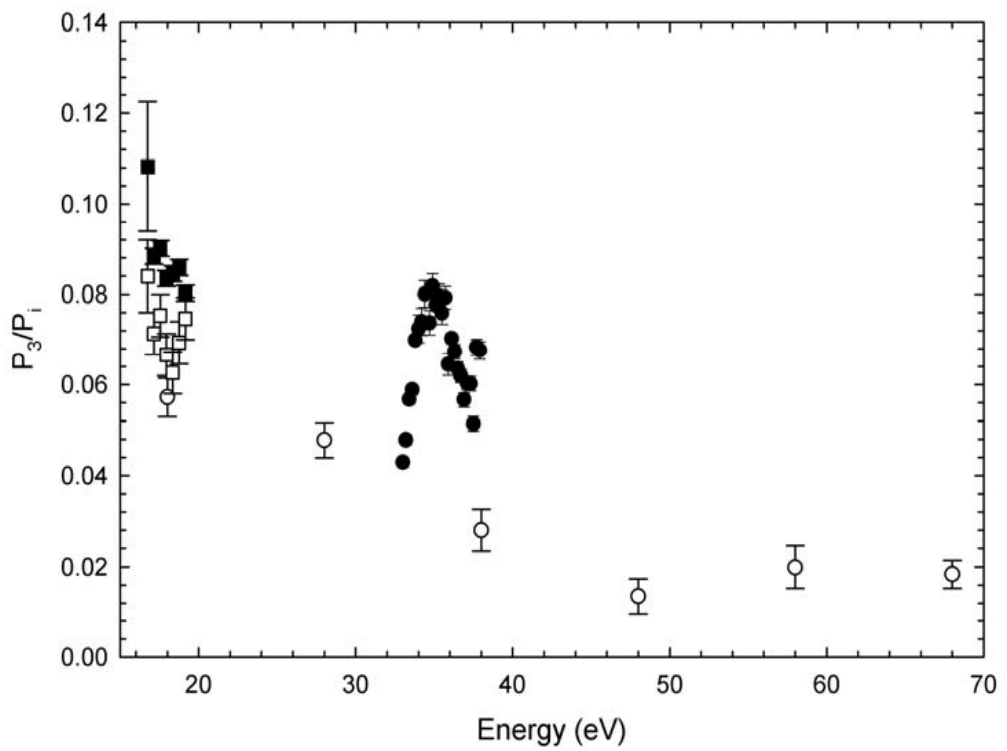

FIGURE 2. $\mathrm{P}_{3}$ of $\mathrm{H} \alpha(656 \mathrm{~nm})$ atomic fluorescence, normalized to the incident photon or electron polarization $\mathrm{P}_{\mathrm{i}}$, vs. incident electron or photon energy. Photon results are also divided by two to take into account the different angular momentum deposited by electrons vs. photons. Solid and open squares: electron data of ref. 13 with different incident electron polarizations; open circles: electron data of ref. 12; solid circles: photon data of this work. 
have measured $\mathrm{P}_{3}$ of $\mathrm{H}(\mathrm{n}=3), \mathrm{H} \alpha(656 \mathrm{~nm})$ fluorescence resulting from electronimpact dissociation of $\mathrm{H}_{2}[12,13]$. These data are shown in Figure 2. Not surprisingly, significant polarizations near the $n=3$ production threshold of $\sim 17 \mathrm{eV}$ are apparent in these data, with a slow drop off as the electron energy increases.

It is instructive to compare these polarization values with those resulting from the direct impact excitation of atoms by polarized electrons. To do this, we define a "spin transfer efficiency," $T$, equal to the initial spin polarization of the excited system divided by the electron spin polarization, $\mathrm{P}_{\mathrm{e}}$. Thus, if we excited a pure molecular triplet state by exchange, $T=2 / 3[1]$. Following dissociation, we assume that the atomic $3 \mathrm{~s}, 3 \mathrm{p}$, and $3 \mathrm{~d}$ states all have equal spin polarization $T$, , and that this polarization is completely coupled to the orbital angular momentum in the case of the $3 \mathrm{p}$ and $3 \mathrm{~d}$ states, while we ignore hyperfine depolarization [12]. Taking into account the branching ratio between $\mathrm{H} \alpha$ and Ly $\alpha$ radiation (see Figure 3), assuming that light from the $3 \mathrm{~s}$ state is unpolarized, and using published data for the relative production cross sections for $3 \mathrm{~s}, 3 \mathrm{p}$, and $3 \mathrm{~d}$ states as the result of electron impact dissociation of $\mathrm{H}_{2}$ [14], we can infer a threshold value of $T^{\prime}=0.37$. Assuming that all three $\mathrm{n}=3$ initial populations are equal, $T^{\prime}=0.47$. These values are surprisingly comparable to those for direct excitation of, e.g., alkali atoms, from $\mathrm{Na}(T=0.25)$ to $\mathrm{Cs}(T=0.45)$ [15].

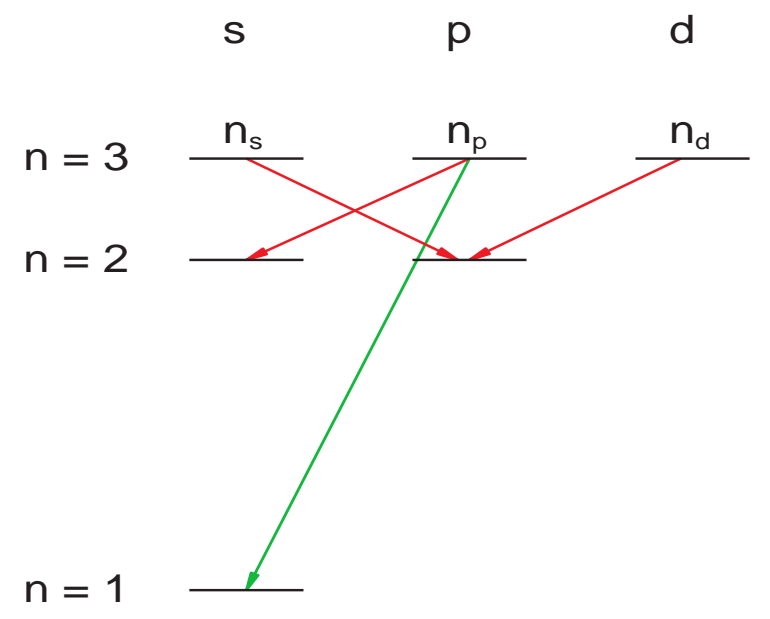

FIGURE 3. Grotrian diagram of the first three energy levels of the $\mathrm{H}$ atom. The circular polarization we observe is due to transitions from the $3 \mathrm{p}$ and $3 \mathrm{~d}$ states; photons from the $3 \mathrm{~s}$ state are unpolarized. In the calculations of T', initial populations of the $n=3$ states are taken from ref. 14 . Only $\sim 1 / 7^{\text {th }}$ of the $3 p$ states decay to the $2 \mathrm{~s}$ state; the rest decay via Lya emission. 


\section{EXPERIMENTS WITH PHOTONS}

We can learn more about how angular momentum is distributed in a dissociating molecular complex by using circularly-polarized incident photons to provide the dissociation energy. This method has the advantage that a full $\hbar$ of angular momentum is dumped into the target, as opposed to $\hbar / 2$ for electrons. Moreover, spinorbit coupling is not needed to convert spin to orbital orientation; the coupling between the photon and the orbital angular momentum of the molecule is direct. In a recent experiment done at the Advanced Light Source (ALS) at Lawrence Berkeley Laboratory, we have measured $\mathrm{H} \alpha \mathrm{P}_{3}$ values when light with energy between 33 and $38 \mathrm{eV}$ was used to dissociate $\mathrm{H}_{2}$. The linearly-polarized synchrotron radiation was turned into circularly-polarized light by passage through a four-reflection quarterwave retarder [2]. These data are shown in Figure 2. They have been divided by two to account for the larger amount of angular momentum carried by the photons, and adjusted to correspond to photon emission directly along the incident photon axis. Interestingly, the $\mathrm{P}_{3}$ values produced by the incident photons are comparable to the results for electron bombardment, even though the angular momentum coupling is much more efficient. It is important to keep in mind, however, that the internal molecular spin-orbit coupling, while serving to produce orbital orientation in the case of electron bombardment, acts only to reduce it (by spinning up the electrons at the expense of orbital angular momentum) in the case of photon bombardment.

\section{POLARIZED MOLECULAR FLUORESCENCE}

Upon closer examination, the simple model of rotational destruction of polarization for excited molecular fluorescence fails. We used a broad $(600 \pm 5 \mathrm{~nm})$ interference filter to monitor fluorescence in the Fulcher band of $\mathrm{H}_{2}$ excited by polarized electron impact. These results are shown in Figure 1 together with the $\mathrm{N}_{2}$ and $\mathrm{N}_{2}{ }^{+}$data. Our

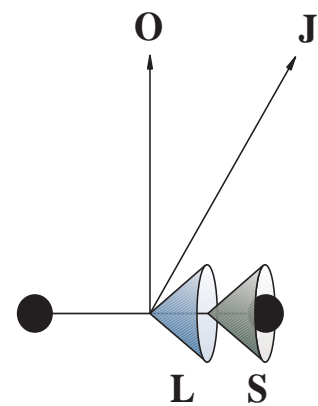

(a)

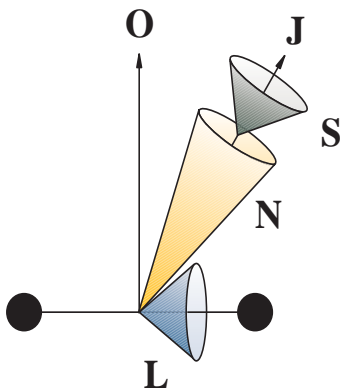

(b)

FIGURE 4. Hund's cases a) and b). The nuclear rotational angular momentum is $\mathbf{O}$; total molecular angular momentum $=\mathbf{J}$. In the case of $\mathrm{H}_{2}, \mathbf{L}+\mathbf{O}=\mathbf{N}$. 
$600 \mathrm{~nm}$ filter passes light which is due to a variety of $\mathrm{H}_{2}$ transitions; we estimate that approximately $94 \%$ of the transmitted intensity is due to emission by triplet states which can, in principle, produce circularly-polarized light. Naively, one might expect that $\mathrm{H}_{2}$ fluorescence would be suppressed even more than that of $\mathrm{N}_{2}$, since, classically speaking, the $\mathrm{H}_{2}$ molecule at a given temperature rotates somewhat more rapidly than does a nitrogen molecule, while both have comparable fluorescence lifetimes. This neglects the relative strength of the couplings between the various angular momenta in the molecule, however. Nitrogen is a Hund's case a) molecule, in which the spin is essentially coupled directly to the internuclear axis (see Figure 4). This is because the spin-orbit coupling time is comparable to the internuclear rotational period, $\sim 10^{-13} \mathrm{~s}$. Hydrogen, on the other hand, is a Hund's case b) molecule, meaning that the spin is much more loosely tied to the internuclear axis. While its rotational period is still $\sim 10^{-13} \mathrm{~s}$, its spin-orbit coupling time is closer to $\sim 10^{-10} \mathrm{~s}$. Thus hydrogen can retain a better "memory" of the initial spin direction [12]. We have investigated several other transitions in molecular hydrogen, and find them to be polarized as well, although with generally lower polarization than that shown in Figure 1. Clearly a systematic study of this problem, with wavelength selection for specific vibrational and rotational levels is warranted.

\section{ACKNOWLEDGMENTS}

We would like to thank Kevin Dooley, Orhan Yenen, and Duane Jaecks for useful conversations and contributions to the photodissociation data reported here. This work has been funded by the U.S. Department of Energy through use of the ALS, and the US National Science Foundation Grants PHY-0354946 (TJG), and PHY-0321055 (KWM). One of us (JEF) wishes to acknowledge support from the University of Newcastle Outside Studies Program.

\section{REFERENCES}

1. K. Blum, Density Matrix Theory and Applications, 2nd ed. (Plenum, New York, 1996).

2. K.W. McLaughlin, O. Yenen, D.H. Jaecks, T.J. Gay, M.M Sant'Anna, D. Calabrese, and B. Jordan-Thaden, Phys. Rev. Lett. 88, 123003 (2002).

3. J.W.McConkey, S.Trajmar, J.C.Nickel, and G.Csanak, J. Phys. B 19, 2377-2392 (1986).

4. M.A.Khakoo and J.W.McConkey, J. Phys. B 20, L175-L179 (1987).

5. J.A.Guest, K.H.Jackson, and R.N.Zare, Phys.Rev.A 28, 2217-2228 (1983).

6. E.Flemming, O.Wilhelmi, H.Schmoranzer, and M.Glass-Maujean, J.Chem.Phys. 103, 4090-4096 (1995).

7. T.P.Rakitzis and R.N.Zare, J.Chem.Phys. 110, 3341-3350 (1999).

8. K.J.Goecke, J.Kessler, and G.F.Hanne, Phys. Rev. Lett. 59, 1413-1415 (1987).

9. M.Eminyan and G.Lampel, Phys. Rev. Lett. 45, 1171-1174 (1980).

10. T.J.Gay, J.E.Furst, K.W.Trantham, and W.M.K.P.Wijayaratna, Phys. Rev. A 53, 1623-1629 (1996).

11. G.F.Hanne in Novel Aspects of Electron-Molecule Collisions,K.Becker ed. (World Scientific, Singapore, 1998).

12. A.S. Green, G.A. Gallup, M.A. Rosenberry, and T.J. Gay, Phys. Rev. Lett. 92, 093201 (2004).

13. J.F.Williams and D.H. Yu, Phys. Rev. Lett. 93, 073201 (2004).

14. W. Kedzierski, A. Abdellatif, J.W. McConkey, K. Bartschat, D.V. Fursa, and I. Bray, J. Phys. B 34, 3367-3375 (2001).

15. C.P. Na $\beta$, M. Eller, N. Ludwig, E. Reichert, and M.Webersinke, Z. Phys. D 11, 71-80 (1989). 